\title{
Real-time capillary convective PCR based on horizontal thermal convection
}

\author{
Xianbo Qiu ${ }^{1}$ Jung II Shu ${ }^{2} \cdot$ Oktay Baysal ${ }^{2} \cdot J \mathrm{Wu}^{1} \cdot$ Shizhi Qian $^{2} \cdot$ Shengxiang $\mathrm{Ge}^{3} \cdot \mathrm{Ke} \mathrm{Li}^{4} \cdot$ Xiangzhong Ye $^{4}$. \\ Ningshao Xia ${ }^{3} \cdot$ Duli Yu ${ }^{1,5}$
}

Received: 13 August 2018 / Accepted: 8 February 2019 / Published online: 18 February 2019

(c) Springer-Verlag GmbH Germany, part of Springer Nature 2019

\begin{abstract}
A real-time, capillary, convective polymerase chain reaction (PCR) system based on horizontal convection is developed and analyzed. The capillary tube reactor is heated at one end in a pseudo-isothermal manner to achieve efficient thermal cycling based on horizontal thermal convection. Mathematical modeling and the in silico simulations indicate that, once consistent temperature gradient along the horizontal capillary tube has been established, a repeatable and continuous circulatory flow in the horizontal directions is created. The formed convection is able to transport PCR reagents through different temperature zones inside the horizontal capillary tube for different reaction stages, that is, DNA denaturing, annealing, and extension in a typical PCR cycle. Furthermore, the effectiveness and efficiency of the horizontal convection for PCR thermal cycling in a capillary tube is confirmed by experimentation. To evaluate the concept of horizontal convective PCR, a compact system, which is able to heat the capillary tube from one end and monitor the fluorescence in situ with a smartphone camera, is developed for real-time amplification. With horizontal thermal convection, influenza A (H1N1) virus nucleic acid targets with a limit of detection (LOD) of $1.0 \mathrm{TCID}_{50} / \mathrm{mL}$ can be successfully amplified and detected in $30 \mathrm{~min}$, which is promising for efficient nucleic acid analysis in point-of-care testing.
\end{abstract}

Keywords Polymerase chain reaction (PCR) - Capillary convective PCR $\cdot$ Horizontal thermal convection $\cdot$ Real time Point-of-care (POC) testing

This article is part of the topical collection "2018 International Conference of Microfluidics, Nanofluidics and Lab-on-a-Chip, Beijing, China”, guest edited by Guoqing Hu, Ting Si and Zhaomiao Liu.

Xianbo Qiu

xbqiu@mail.buct.edu.cn

1 Institute of Microfluidic Chip Development in Biomedical Engineering, College of Information Science and Technology, Beijing University of Chemical Technology, Beijing 100029, China

2 Institute of Micro/Nanotechnology, Old Dominion University, Norfolk, VA 23529, USA

3 National Institute of Diagnostics and Vaccine Development in Infectious Diseases, Xiamen University, Xiamen 361005, China

4 Beijing Wantai Biological Pharmacy Enterprise Co. Ltd, Beijing 102206, China

5 Beijing Advanced Innovation Center for Soft Matter Science and Engineering, Beijing 100029, China

\section{Introduction}

Polymerase chain reaction (PCR) is a method that enables templates of nucleic acid to be amplified at the rate of exponential growth. This technique is widely used in criminal forensics, organism detection, and applications that require nucleic acid identifications. Its fundamentals are based on the different temperature-dependent reactions, which are PCR thermal cycling processes including denaturation, annealing, and extension. In the denaturation stage, a double-stranded DNA melts at around $95^{\circ} \mathrm{C}$, splitting into two single-stranded DNAs. They cool down at around $55^{\circ} \mathrm{C}$ in the annealing stage; primers are allowed to complement each of the single-stranded DNA templates at this temperature. In the extension stage, the two single-stranded DNAs are synthesized by the aid of enzymes at around $72{ }^{\circ} \mathrm{C}$, resulting in two new double-stranded DNAs (Qiu et al. 2010).

In principle, PCR requires precise and repetitive thermal cycling to perform different reaction stages including denaturing, annealing, and extension, which cause the significantly 
long amplification time to be contributed by both transition time and dwelling time between or inside each stage (Qiu et al. 2010). Obviously, microfluidic PCR provides potentiality to reduce PCR amplification time by significantly decreasing the reagent volume, adopting new heating strategies, or using new substrate for reaction chamber (Bian et al. 2015; Sposito et al. 2016; Houssin et al. 2016). It has been proven that a significantly high ramping rate can be achieved when a microfluidic PCR chamber with a smaller/reduced size is heated efficiently (Son et al. 2015). Currently, for most of commercially available PCR systems, whether normal PCR or real-time PCR, PCR reaction with $25-50 \mu \mathrm{L}$ size is usually performed in a traditional $200 \mu \mathrm{L}$ tube. Because of the advantages of microfluidic PCR, microfluidic PCR systems can improve the critical performance of PCR, for example, to reduce the required time, improve efficiency, simplify the procedure, and lower down the cost.

However, there have been at least two disadvantages for microfluidic PCR with such a small reaction chamber. One is that the smaller/reduced size reactor could limit the detection sensitivity, since a relatively low number of nucleic acid templates could be utilized. The other is that PCR thermal cycling requires a complicated and elaborate temperature control system for repeatedly switched heating and cooling. Even with the inconvenience for primer design and the difficulty for quantitative detection, because of both the simplified heating format and the satisfied sensitivity with reasonably short amplification time, various isothermal amplification methods, for example, LAMP, RPA, RCA, and NASBA, have exhibited their advantages for nucleic acid analysis in point-of-care testing (Craw and Balachandran 2012; Liu et al. 2011; Liao et al. 2016).

To achieve efficient PCR thermal cycling, even with a simplified heating format, for example, in a pseudo-isothermal manner, convective PCR was invented by introducing stable thermal convection within a capillary tube reactor with a proper steady-state temperature gradient between its two ends (Krishnan et al. 2002; Priye et al. 2016; Chen et al. 2004; Li et al. 2016). When the capillary tube is heated with one or two properly set temperatures at one or two ends, a continuous and consistent circulation of reactants between the hot $\left(\sim 95^{\circ} \mathrm{C}\right)$ and cool zones $\left(\sim 55^{\circ} \mathrm{C}\right)$ of the capillary tube is induced for spatial PCR thermal cycling without active pumping. That is, the spatially different temperature causes the density to vary, and the buoyant force drives the flow circulation. As PCR reagents circulate in the different temperature zones of the capillary tube, all of the PCR thermal reaction stages, such as denaturation, annealing, and extension, take place in a one-flow cycle; therefore, the reactions are affected by flow velocity. Different from both existing microfluidic PCRs and isothermal amplification methods, convective PCR is capable of achieving significantly more efficient thermal cycling based on a simple setup. This is true even with a large reaction size, such as up to tens of microliters, which is highly desired for point-of-care testing. Table 1 shows a brief summary of the currently existing PCR systems including traditional PCR, microfluidic PCR, and convective PCR.

Previously, we have presented different convective PCR systems with different detection formats or different heating mechanisms (Qiu et al. 2016, 2017a, b, c). For each implementation, a capillary tube, sitting vertically, is heated from both the bottom and the top or just from the bottom to achieve an efficient convective PCR amplification. Here, in another way, we report real-time convective PCR based on horizontal thermal convection with a laid down capillary reactor. The inherent mechanism of horizontal convective PCR is analyzed with mathematical modeling for in silico simulations and confirmed with experimentation. A compact setup is developed to facilitate effective heating with a fixed temperature as well as real-time fluorescence monitoring with a smartphone. The performance of the horizontal convective PCR is evaluated with the detection to influenza $A$ (H1N1) virus nucleic acid targets.

The rest of the paper is organized into four sections: "Real-time horizontal convective PCR" for the description of the horizontal CPCR system, "Three-dimensional mathematical model and in silico simulations" for the description of system modeling, :Results and discussion" for the description of performance evaluation, and "Conclusions".

\section{Real-time horizontal convective PCR}

\subsection{Horizontal convective PCR}

As shown in Fig. 1, the capillary tube reactor with smooth inside surface has a total reaction size of $\sim 40 \mu \mathrm{L}$ with an outside diameter of $3.3 \mathrm{~mm}$, an inside diameter of $1.7 \mathrm{~mm}$, and a length of $19 \mathrm{~mm}$. Capillary tubes are made from polycarbonate by injection molding to ensure a remarkable smoothness to reduce undesired surface absorbing as well as satisfy transparency for optical detection (Fig. 1).
Table 1 A brief summary of the currently existing PCR systems

\begin{tabular}{lll}
\hline Name & Reactor & Characteristics (volume, heating mode, time) \\
\hline Traditional PCR & PCR tube & $25-50 \mu \mathrm{L}$, thermal cycling, 1-2 h \\
Microfluidic PCR & Customized chip & nl- $\mu \mathrm{L}$, thermal cycling, a few minutes to hours \\
Convective PCR & Capillary tube & $25-50 \mu \mathrm{L}$, pseudo-isothermal, $\leq 30$ min \\
\hline
\end{tabular}




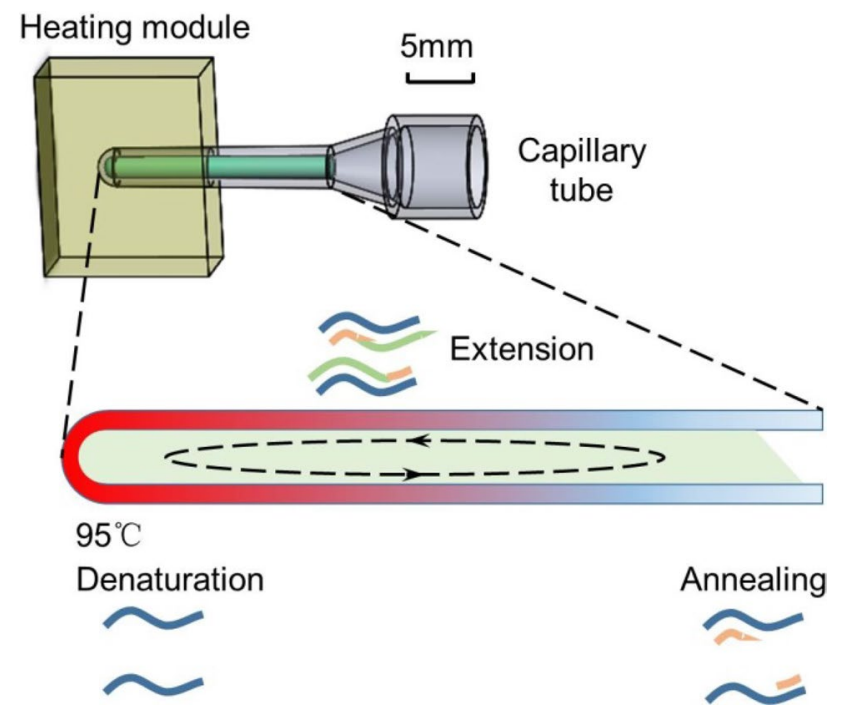

Fig. 1 Capillary tube PCR reactor for horizontal convective amplification

As shown in Fig. 1, different from our previous convective PCR systems, here the capillary tube is laid down to achieve horizontal thermal convection. Once the horizontal capillary tube is heated from one end with a fixed temperature to generate a stable steady-state temperature gradient along the horizontal directions, a continuous back-and-forth circulation of reactants between hot and cool zones of the horizontal reactor is induced to facilitate efficient PCR thermal cycling.

\subsection{Real-time horizontal convective PCR system}

For proof of concept, a compact setup was developed to perform real-time horizontal convective PCR. As shown in Fig. 2, a single-end heating strategy is adopted to heat the capillary tube from one end with a resistive heater. A thermistor is applied for temperature measurements. Meanwhile, fluorescence detection with a smartphone camera is performed for real-time monitoring of convective amplification in situ.

As shown in Fig. 2, two sub-systems, respectively, for the thermal control and the optical detection are developed and integrated to implement real-time horizontal convective PCR. A PID control loop is developed to ensure precise temperature control for single-end heating. An optical module, which consisted of an LED coupled with an optical filter for excitation, and a smartphone camera coupled with another optical filter for collection of fluorescence emission, are developed to enable convenient detection.

For the Taqman probe conjugated with FAM ${ }^{\mathrm{TM}}$ used in horizontal convective PCR amplification, a specific LED with a proper wavelength around $475 \mathrm{~nm}$ is adopted to achieve high

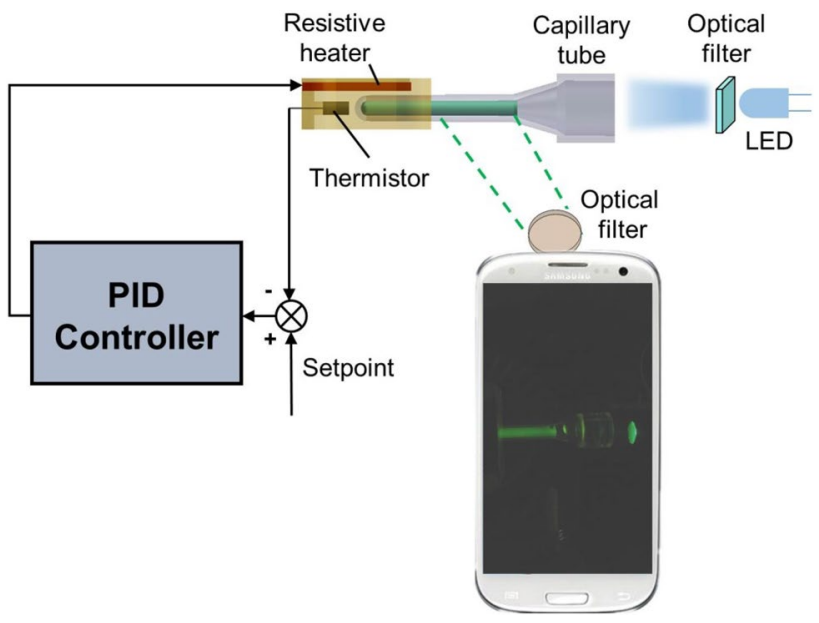

Fig. 2 Schematic depiction of the horizontal convective PCR system

excitation efficiency. A custom Java application program running on the smartphone controls the camera to take fluorescent images from the horizontal capillary tube according to a proper time interval. For quantification, the collected fluorescent images are further processed by a custom image analysis algorithm running on the same smartphone to achieve the fluorescence intensity amplitude inside the area of convective amplification.

A compact system $(100 \times 70 \times 150 \mathrm{~mm})$ is developed to perform real-time, horizontal, convective PCR with a total component cost of about \$50, not including the smartphone. A smartphone that performs a multifunction for fluorescence detection, signal analysis, and graphical operation interfacing can nowadays be purchased at a relatively low cost.

\section{Three-dimensional mathematical model and in silico simulations}

A conjugate heat-momentum transfer model is adopted for the present process. The 3-D computational domain has fluid and solid regions as shown in Fig. 3. The capillary tube is made of polycarbonate used as the material for the solid domain. For the fluid domain, we assume that the capillary tube is filled with pure water, and the flow is steady state, laminar for simple computation. The model is dominated by the governing equations for the convection (Eqs. 1-3) in the fluid and the conduction (Eq. 4) in the solid (White 2011).

$\nabla \cdot(\rho \vec{u})=0$,

$\rho(\vec{u} \cdot \nabla) \vec{u}=-\nabla p+\rho \vec{g}+\mu \nabla^{2} \vec{u}$,

$\rho C_{p}\left(\vec{u} \cdot \nabla T_{\mathrm{f}}\right)=\nabla \cdot\left(k_{\mathrm{f}} \nabla T_{\mathrm{f}}\right)$,

$\nabla \cdot\left(k_{\mathrm{s}} \nabla T_{\mathrm{s}}\right)=0$ 


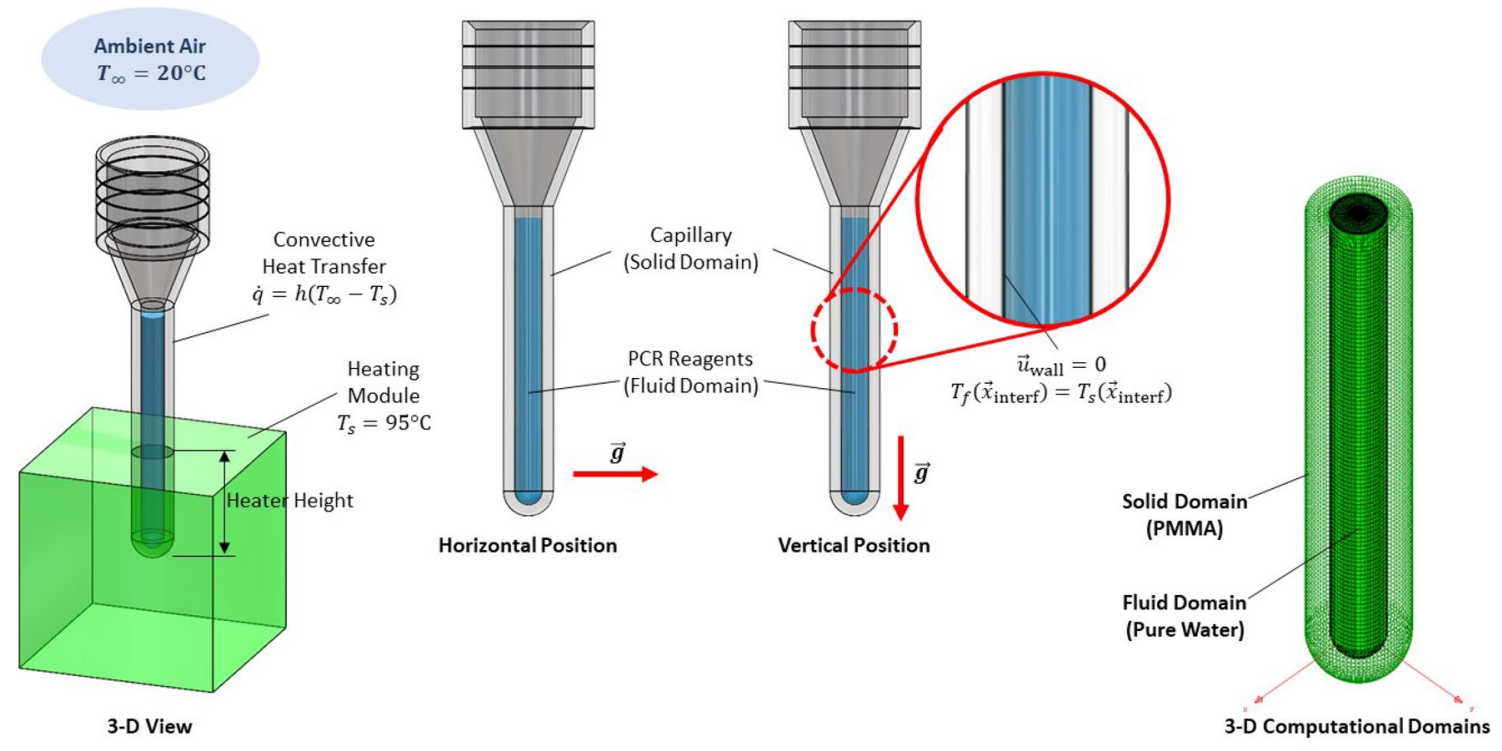

Fig. 3 3-D model for the capillary tube, boundary conditions, and its computational domains

where $\rho(T)$ is the fluid density, determined based on the temperature; $\vec{u}$ is the flow velocity; $p$ denotes the pressure; $\vec{g}$ represents the gravitational acceleration; $\mu(T)$ is the dynamic viscosity of fluid, also determined based on the temperature; $C_{p}(T)$ is the isobaric specific heat of fluid depending on the temperature; $T_{\mathrm{f}}$ denotes the fluid temperature; $k_{\mathrm{f}}(T)$ is the temperature-dependent thermal conductivity of the fluid; $k_{\mathrm{s}}$ is the thermal conductivity of the solid; and finally $T_{\mathrm{s}}$ denotes the temperature on the solid.

A polynomial curve fit for the thermal properties of the liquid is developed and used, specifically for the convection due to the density variation (Cengel and Boles 2014). For the heat conduction in the solid domain, however, constant values of thermal properties are utilized. As depicted in Fig. 3, the boundary conditions are applied as follows:

1) No-slip condition $\left(\vec{u}_{\text {wall }}=0\right)$ for velocity is applied on the solid boundaries (Cengel and Boles 2014).

2) The temperature is set to $95^{\circ} \mathrm{C}$ on the outer surface of the capillary tube where the heater is located.

3) For the convective heat transfer between the solid and the ambient air, $\dot{q}=h\left(T_{\infty}-T_{\mathrm{s}}\right)$, the convective heat transfer coefficients of the horizontal and vertical positions are set to $11.5 \mathrm{~W} /\left(\mathrm{m}^{2} \mathrm{~K}\right)$ (Cengel and Boles 2014; Holman 2010; Churchill and Chu 1975), and 26.7 $\mathrm{W} /\left(\mathrm{m}^{2} \mathrm{~K}\right)$ (Boetcher 2014), respectively, as a nominal value for the free convection of air. Note that the free space for the vertical configuration is larger than that for the horizontal case, yielding higher convective heat transfer coefficient for the vertical position.

4) The temperature is always continuous at the contact area of the two bodies, $T_{\mathrm{f}}\left(\vec{x}_{\text {interf }}\right)=T_{\mathrm{s}}\left(\vec{x}_{\text {interf }}\right)$, and the heat flux is the same on both sides of the fluid-solid interface (Cengel and Boles 2014).

For the spatial discretization, structured, multi-domain meshes are generated for both the fluid and the solid regions. The discretized equations for the steady-state problem are then solved using the SIMPLE algorithm. Our OpenFOAM-based in-house computer code is run for each case until the numerical residuals of velocity and temperature are less than or equal to the pre-specified tolerances, i.e., 1e-8 (Greenshields 2017). Mesh-independent study was conducted to ensure that the results converged and were independent of meshes. The results are mesh independent when the fluid and solid domains comprise 224,576 and 123,948 cells, respectively (Shu et al. 2019).

\section{Results and discussion}

Based on prior experience, the heating temperature for horizontal convective PCR should be set to about $95{ }^{\circ} \mathrm{C}$ when the capillary tube is heated from its dead end. Witnessing the difference between the controlled temperature from the heating block and the reaction temperature from the capillary tube, the setpoint for the feedback temperature control system is intentionally adjusted for temperature compensation. With a properly tuned PID controller, the heating temperature can be consistently maintained with a steady-state error of $\pm 0.1{ }^{\circ} \mathrm{C}$, which ensures the amplification efficiency of horizontal convective PCR. 


\subsection{Detection of influenza virus}

Influenza A (H1N1) virus nucleic acid targets are detected to evaluate the performance of horizontal convective PCR. Meanwhile, the performance of the horizontal convective PCR is compared with that of vertical convective PCR for validation. Diluted influenza A virus culture stock samples $\left(1.0 \mathrm{TCID}_{50} / \mathrm{mL}\right)$ with negative control (NC) were respectively tested.

The convective PCR reaction mix comprises $3 \mu \mathrm{L}$ mixture of primers (Beijing Wantai Biological Pharmacy Enterprise, Ltd.) and a Taqman probe which is labeled with $\mathrm{FAM}^{\mathrm{TM}}$ at $5^{\prime}$ end and Eclipse at the 3' end, $4 \mathrm{mM}$ dNTP and $4 \mu \mathrm{L}$ of Fast Buffer I (Mg ion buffer) (all Takara Bio Inc., Shiga, Japan), $0.4 \mathrm{U}$ of AMV reverse transcriptase (Promega, USA), $1 \mathrm{U}$ of SpeedSTAR HS DNA polymerase (Takara), $10 \mu \mathrm{L}$ of the purified influenza A (H1N1) RNA as per the above protocol, and molecular-biology-grade water to a total reaction volume of $40 \mu \mathrm{L}$. The primer sequence used with influenza H1N1 template is expected to yield a 105-bp amplicon. After loading the PCR reaction mixture, the reaction is sealed with a layer of $10 \mu \mathrm{L}$ sterile mineral oil (Sigma-Aldrich, St. Louis, MO, USA). Because of the high viscosity of the mineral oil, it stays on the right position without sealing, partly due to the surface tension inside the capillary tube.

The production of amplicons with Taqman probes labeled with FAM $^{\mathrm{TM}}$ is monitored in real time by a smartphone camera. Furthermore, the fluorescence intensity amplitude of each image is extracted and analyzed with custom software. As shown in Fig. 4, the real-time fluorescence intensity curves for horizontal convective PCR (Fig. 4a) saturate after approximately $25 \mathrm{~min}$, which is comparable to that of the existing vertical convective PCR (Fig. 4b). Compared to the positive test, $\mathrm{NC}$ did not show significant increase on fluorescence signal intensity in convective amplification (data not shown here). The experiments were repeated for at least three times and similar results were obtained.

As shown in Fig. 4, an efficient convective PCR amplification, similar to vertical convective PCR, is achieved when the capillary tube is positioned horizontally. As a proof of concept, nucleic acid targets from H1N1 sample with a concentration of $1.0 \mathrm{TCID}_{50} \mathrm{ml}$ (limit of detection) can be successfully detected. Similar to the existing vertical convective PCR, it takes significantly less time for the horizontal convective PCR to perform nucleic acid amplification with a reasonable sensitivity compared to the traditional PCR. As shown in Fig. 4, the two curves for the horizontal and vertical PCR, respectively, with the same sample of $1.0 \mathrm{TCID}_{50} /$ $\mathrm{mL}$, are different in both intensity and the time they start to rise, because of the uncontrolled cycle number of thermal convection. It has been demonstrated that the horizontal convective PCR is capable of achieving the desired amplification as efficiently as the vertical convective PCR. Hence, this conclusion provides an alternative for the capillary tube reactor to be put horizontally or vertically, whichever better accommodates a specific design. A good example may be a case of an integrated microfluidic chip system for nucleic acid analysis.

\subsubsection{In silico simulations of the horizontal/vertical convective PCR}

Numerical simulations were carried out for the horizontal and vertical convective PCRs and a sample of the results is presented in Fig. 5. The maximum temperatures for both cases approach $\sim 95^{\circ} \mathrm{C}$, which is deemed to be the proper
Fig. 4 Real-time convective PCR with influenza A (H1N1) virus RNA template (1.0 $\mathrm{TCID}_{50} / \mathrm{mL}$ ). Fluorescence images at different amplification times and the corresponding fluorescence signal intensity as a function of time, respectively, for: a horizontal convective PCR and $\mathbf{b}$ vertical convective PCR (a)
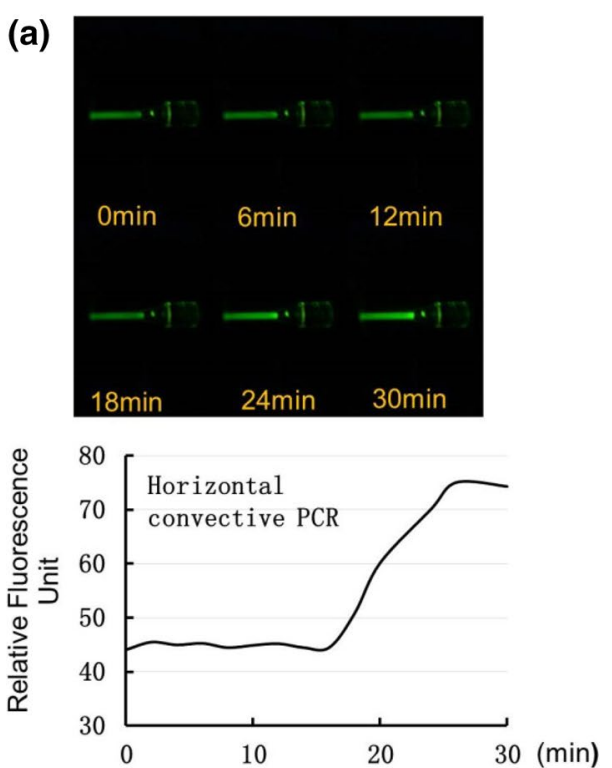

(b)
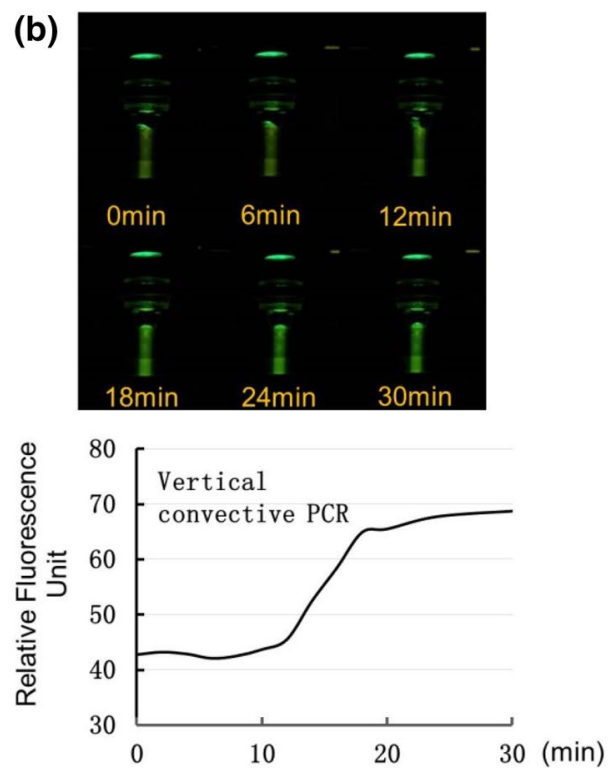
temperature for DNA denaturation; however, there is a stark difference in the obtained minimum temperatures. The temperature at the top remains minimal since flow is naturally cooled, transferring heat to the ambient air. The apropos magnitude of the minimal temperature is approximately $55^{\circ} \mathrm{C}$ for the horizontal position and $\sim 60^{\circ} \mathrm{C}$ for the vertical position, respectively. For the DNA samples used in this experiment, the annealing process occurs in the temperature range of $55-60{ }^{\circ} \mathrm{C}$. It is observed in Fig. 5 that the flow velocity in the vertical position is approximately 1.4 times faster than that of the horizontal case. In both cases, DNA reagents can be circulated from the bottom of the PCR tube to its top and then circulated back to achieve DNA amplification. By comparing Fig. 5c, d, the fluid in the horizontal position is circulated along a perfect oval loop, while in the vertical position, the fluid moves along a slightly twisted oval loop. Due to stronger convective heat transfer, the vertical position has more heat transferred from the bottom to the top, causing the fluid temperature on the top of the tube in the vertical case to be slightly higher than that in the horizontal position. For the same reason, the temperature near the bottom in the vertical position is slightly lower than that in the horizontal position. Figure 6a depicts the fluid temperature along the axis of the tube, and Fig. $6 \mathrm{~b}$ shows the corresponding temperature gradient with respect to the $z$-direction. Beyond a certain point in the $\mathrm{z}$-direction (i.e., $z_{\mathrm{cr}}$ ) which is about half of the total fluid height, the fluid temperature of the vertical position (dashed line) is higher than that of the horizontal position (solid line). In contrast, the fluid temperature of the vertical position is lower than that of the horizontal position in the region of $z<z_{\text {cr }}$ Except in the regions of both ends of the reactor, the magnitude of the temperature gradient in the horizontal position is higher than that of the vertical case, as presented in Fig. 6 b.

The numerical results also qualitatively agree with the experimental results, as shown in Fig. 4. Since the fluid velocity in the vertical position is higher than that in the horizontal position, it takes a shorter time for the fluid to complete one oval loop as shown in Fig. 5. One loop is qualitatively equivalent to one PCR cycle. Therefore, DNA amplification in the vertical position is faster than that in the horizontal case, which explains why the relative fluorescent unit (RFU) signal starts to rise earlier than in the horizontal position due to the fast circulation. However, for sufficiently long time such as $30 \mathrm{~min}$ when the reaction is saturated, the RFU signals for both cases are very close,
Fig. 5 In silico simulations: temperature fields $(\mathrm{K})$ of $\mathbf{a}$ horizontal and $\mathbf{b}$ vertical PCR. Velocity fields $(\mathrm{mm} / \mathrm{s})$ : $\mathbf{c}$ horizontal and d vertical PCR

\section{In-silico simulations of the horizontal/vertical convective PCR}
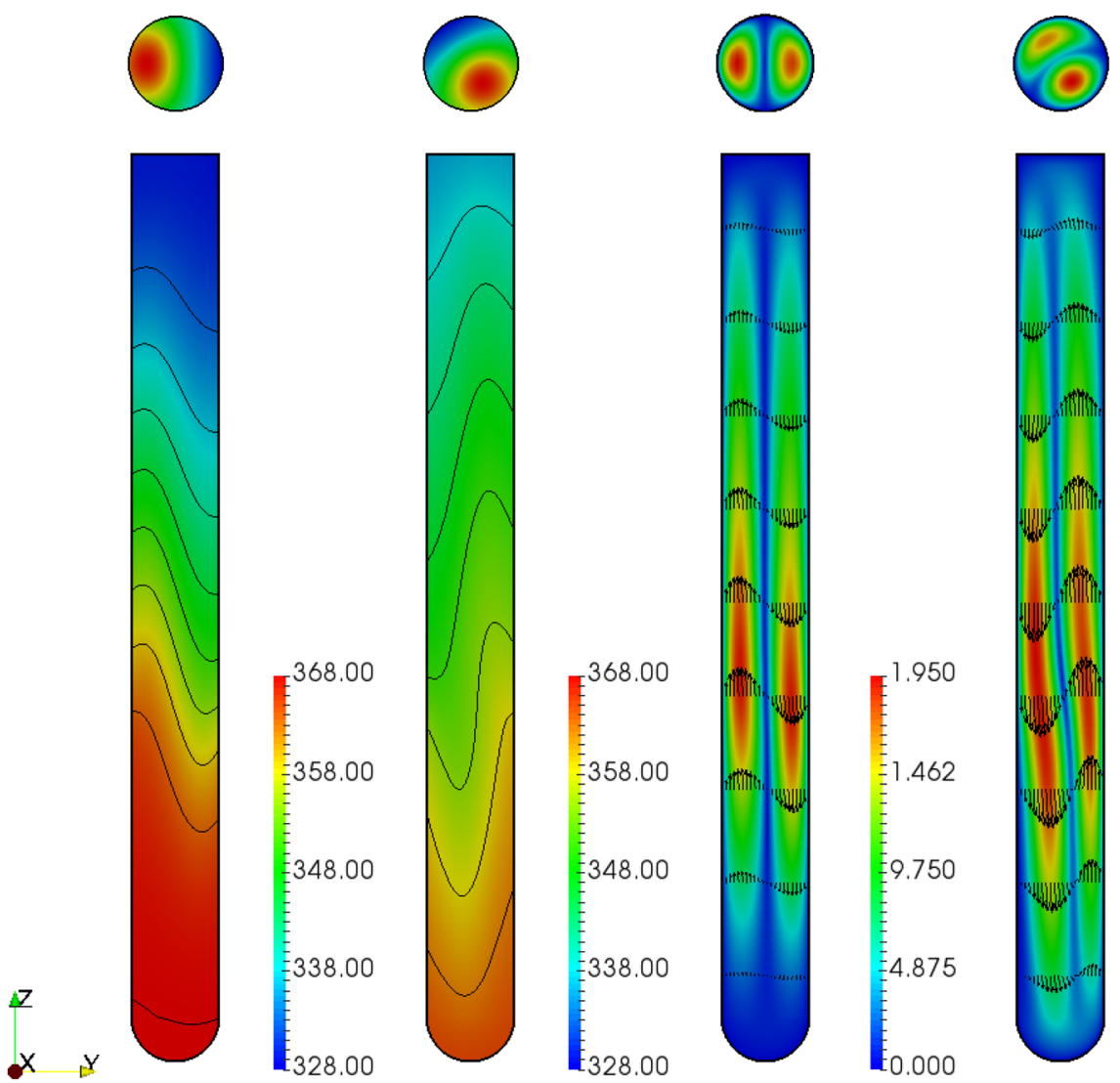

(a)

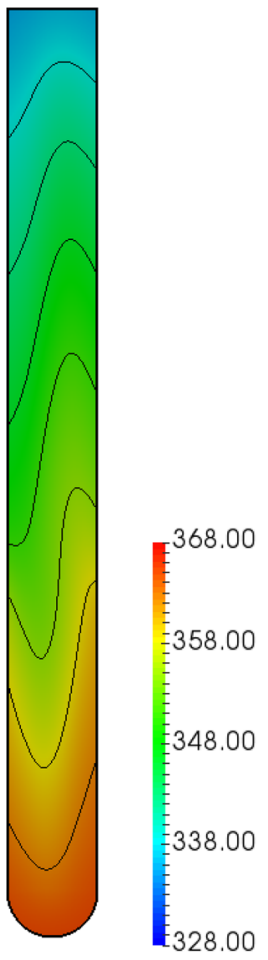

(b)

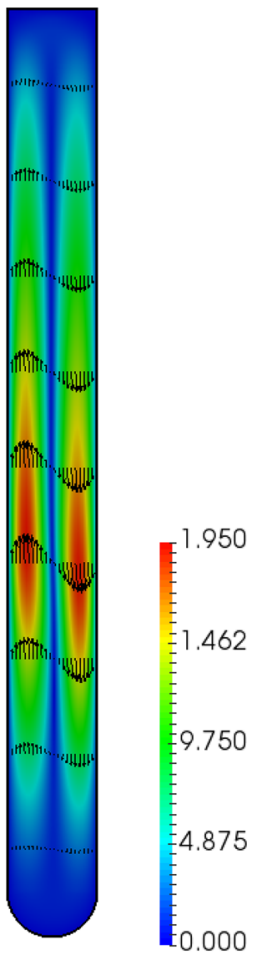

(c)

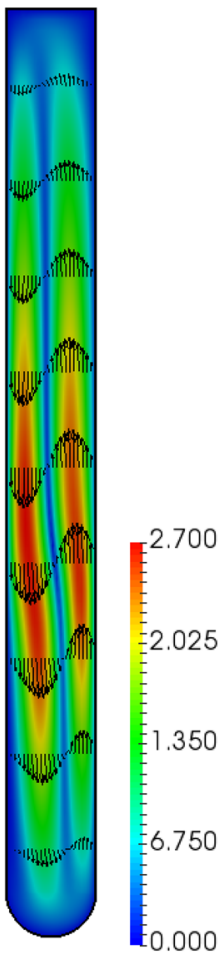

(d) 
Fig. 6 Temperature (a) and temperature gradient $(\mathbf{b})$ with respect to the $z$ direction (a)

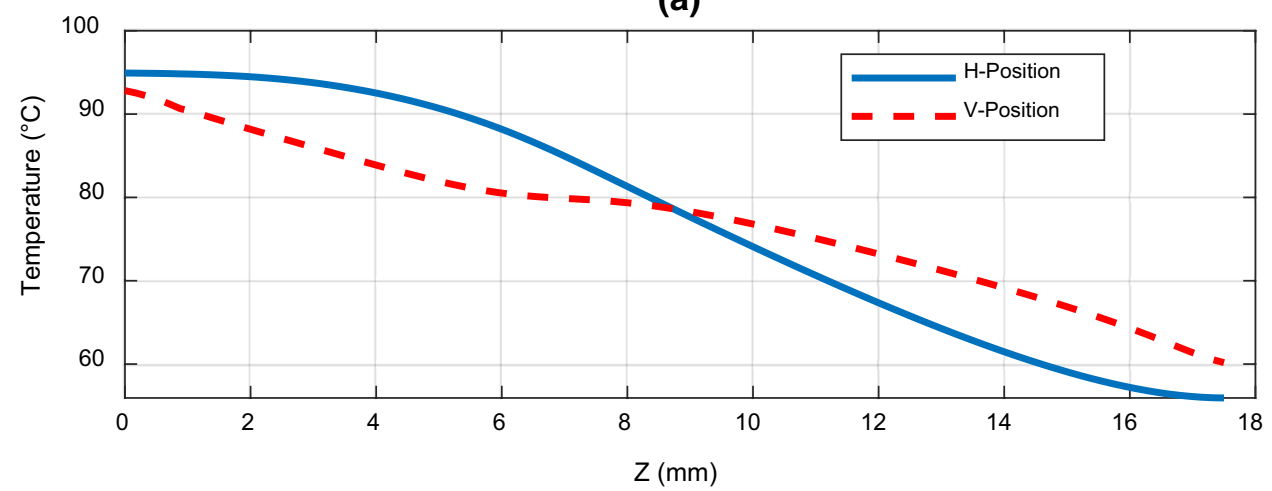

(b)

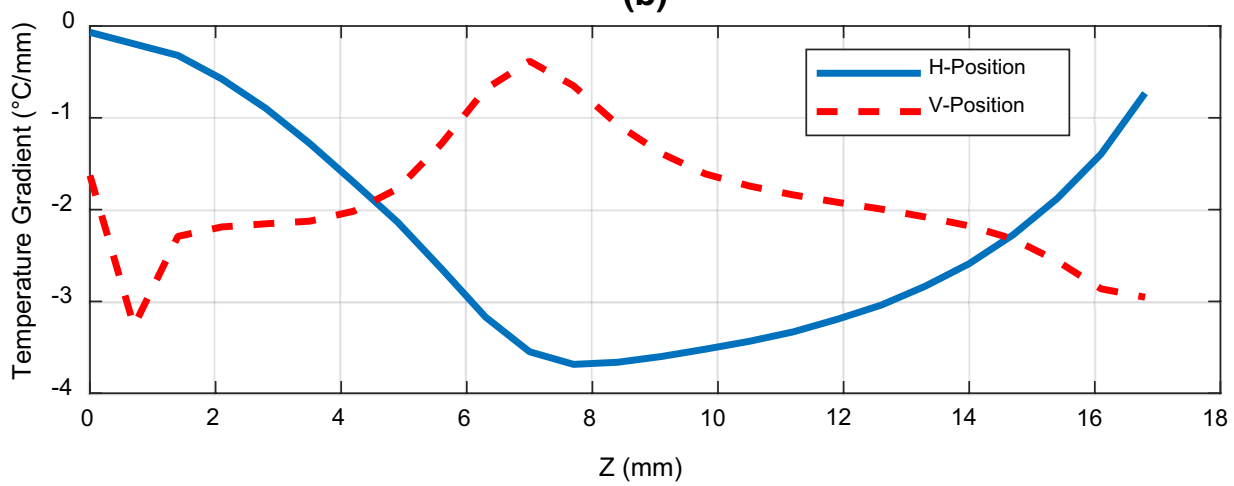

which is approximately 70 and can be evaluated as successful DNA amplification. Although the fluid velocity in the horizontal position is relatively lower than that in the vertical case, lower flow velocity reduces the contribution of convective heat transfer that would help maintain the temperature field effectively for the PCR thermal cyclic processes. The spatial temperature distribution in the vertical position is not the ideal temperature profile for DNA amplification. This partly explains why the RFU signals for both cases are very close in the period of $30 \mathrm{~min}$. It is concluded, therefore, that everything else being equal, the horizontal case would produce almost similar flow circulation as the vertical case. The flow velocity in the horizontal case is slightly lower than that of the vertical case and needs longer time for DNA amplification. However, the temperature distribution in the horizontal position would be more suitable for DNA amplification than the vertical case due to the reduced convection, and this advantage might compensate the aforementioned disadvantage for the horizontal position.

\section{Conclusions}

Different from the existing vertical convective PCR, horizontal convective PCR with a capillary tube reactor has been successfully developed, analyzed, and tested. Similar to the vertical convective PCR, horizontal convective
PCR is capable of significantly reducing the required amplification time with rapid thermal cycling, assisted by thermal convection. A compact system for real-time horizontal convective PCR is developed to perform both a single-end heating and in situ fluorescence monitoring. In addition, a mathematical model and in silico simulations of the horizontal/vertical convective PCR with a capillary tube reactor are developed to analyze in depth its inherent thermo-fluid characteristics. The performance of horizontal convective PCR was evaluated by successfully detecting influenza $A(\mathrm{H} 1 \mathrm{~N} 1)$ virus sample with a concentration of $1.0 \mathrm{TCID}_{50} / \mathrm{mL}$ in less than $30 \mathrm{~min}$. Mathematical modeling of convection PCR was effectively validated by the experimental outcomes. From both the experimental results and the mathematical modeling, it was demonstrated that a horizontal convective PCR was capable of working as efficiently as a vertical convective PCR. The flow velocity in the horizontal PCR is lower than that in the vertical PCR; however, the spatial temperature distribution in the horizontal PCR is more suitable for DNA amplification due to less convective heat transfer. However, the major flow differences between the vertical and horizontal convective PCRs are caused by the different convective heat transfer coefficients occurring in the vertical and horizontal cases. Horizontal convective PCR potentially provides the flexibility to a design process when a convective PCR is a part of an integrated system, 
such as a fully integrated nucleic acid diagnosis system based on microfluidic technology in point-of-care testing.

Funding The present work was supported by the National Natural Science Foundation of China (No. 81871505, 81371711), National Science and Technology Major Project (2018ZX10732101-001-009), the Fundamental Research Funds for the Central Universities (XK1802-4, PYBZ1830), and the research fund to the top scientific and technological innovation team from Beijing University of Chemical Technology (No. buctylkjcx06).

\section{Compliance with ethical standards}

Conflict of interest The authors declare that they have no conflict of interest.

\section{References}

Bian X, Jing F, Li G, Fan X, Jia C, Zhou H, Jin Q, Zhao J (2015) A microfluidic droplet digital PCR for simultaneous detection of pathogenic Escherichia coli O157 and Listeria monocytogenes. Biosens Bioelectron 74(4):770

Boetcher S (2014) Natural convection heat transfer from horizontal cylinders. In: Natural convection from circular cylinders. Springer Briefs in Applied Sciences and Technology. Springer, Cham

Cengel YA, Boles MA (2014) Thermodynamics: an engineering approach, 8th edn. McGraw-Hill Higher Education, New York

Chen Z, Qian S, Abrams WR, Malamud D, Bau HH (2004) Thermosiphon-based PCR reactor: experiment and modeling. Anal Chem 76(13):3707

Churchill S, Chu H (1975) Correlating equations for laminar and turbulent free convection from a vertical plate. Int J Heat Mass Transf 18(9): 1323

Craw P, Balachandran W (2012) Isothermal nucleic acid amplification technologies for point-of-care diagnostics: a critical review. Lab Chip 12(14):2469

Greenshields CJ (2017) OpenFOAM User Guide. OpenFOAM Foundation

Holman J (2010) Heat transfer. McGraw-Hill Higher Education, 10th ed, New York

Houssin T, Cramer J, Grojsman R, Bellahsene L, Colas G, Moulet H, Minnella W, Pannetier J, Leberre M, Plecis A, Chen Y (2016) Ultrafast, sensitive and large-volume on-chip real-time PCR for the molecular diagnosis of bacterial and viral infections. Lab Chip 16(8):1401
Krishnan M, Ugaz VM, Burns MA (2002) PCR in a Rayleigh-Benard convection cell. Science 298:793

Li Z, Zhao Y, Zhang D, Zhuang S, Yamaguchi Y (2016) The development of a portable buoyancy-driven PCR system and its evaluation by capillary electrophoresis. Sens Actuat B Chem 230:779

Liao SC, Peng J, Mauk MG, Awasthi S, Song J, Friedman H, Bau HH, Liu C (2016) Smart cup: a minimally-instrumented, smartphonebased point-of-care molecular diagnostic device. Sen Actuat B Chem 229:232

Liu C, Geva E, Mauk MG, Qiu X, Abrams WR, Malamud D, Curtis K, Owen SM, Bau HH (2011) An isothermal amplification reactor with an integrated isolation membrane for point-of-care detection of infectious diseases. Analyst 136(10):2069

Priye A, Wong S, Bi Y, Carpio M, Chang J, Coen M, Cope D, Harris J, Johnson J, Keller A, Lim R, Lu S, Millard A, Pangelinan A, Patel N, Smith L, Chan K, Ugaz VM (2016) Lab-on-a-drone: toward pinpoint deployment of smartphone-enabled nucleic acid-based diagnostics for mobile health care. Anal Chem 88(9):4651

Qiu X, Mauk MG, Chen D, Liu C, Bau HH (2010) A large volume, portable, real-time PCR reactor. Lab Chip 10(22):3170

Qiu X, Ge S, Gao P, Li K, Yang S, Zhang S, Ye X, Xia N, Qian S (2016) A smartphone-based point-of-care diagnosis of H1N1 with microfluidic convection PCR. Microsyst Technol 23(7):1

Qiu X, Ge S, Gao P, Li K, Yang Y, Zhang S, Ye X, Xia N (2017a) A low-cost and fast real-time PCR system based on capillary convection. J Lab Auto 22(1):13

Qiu X, Zhang S, Xiang F, Wu D, Guo M, Ge S, Li K, Ye X, Xia N, Qian S (2017b) Instrument-free point-of-care molecular diagnosis of H1N1 based on microfluidic convective PCR. Sens Actuat B Chem 243:738

Qiu X, Zhang S, Mei L, Wu D, Guo Q, Li K, Ge S, Ye X, Xia N, Mauk MG (2017c) Characterization and analysis of real-time capillary convective PCR toward commercialization. Biomicrofluidics 11(2):024103

Shu J, Baysal O, Qian S, Qiu X, Wang F (2019) Performance of convective polymerase chain reaction by doubling time. Int $J$ Heat Mass Transf 133:1230

Son JH, Cho B, Hong SG, Sang HL, Hoxha O, Haack AJ, Lee LP (2015) Ultrafast photonic PCR. LIGHT SCI APPL 4(7):e280

Sposito A, Hoang V, Devoe DL (2016) Rapid real-time PCR and high resolution melt analysis in a self-filling thermoplastic chip. Lab Chip 16(18):3524

White F (2011) Fluid mechanics. McGraw-Hill Higher Education, 7th ed, New York

Publisher's Note Springer Nature remains neutral with regard to jurisdictional claims in published maps and institutional affiliations. 